\title{
THE PERMANENT AT A MINIMUM ON CERTAIN CLASSES OF DOUBLY STOCHASTIC MATRICES ${ }^{1}$
}

\author{
BY MARK BLONDEAU HEDRICK
}

\author{
Communicated by Olga Taussky Todd, March 18, 1994
}

\begin{abstract}
Both David London and Mark B. Hedrick have independently generalized a result of $\mathbf{M}$. Marcus and $\mathbf{M}$. Newman concerning the behavior of the permanent at a minimum on the set of doubly stochastic matrices. The author generalizes this last result and simplifies the proof appreciably. He proves the following. Let $A$ be a doubly stochastic matrix and let $X$ be a set of doubly stochastic matrices with the same $(0,1)$-pattern as $A$ in some neighborhood of $A$. If $A$ is a critical point of the permanent relative to $X$, then per $A=\operatorname{per} A(i \mid j)$ for each positive $a_{i j}$.
\end{abstract}

In 1926 [8], B. L. van der Waerden conjectured that the permanent achieves a unique minimum on the set $D_{n}$ of $n \times n$ doubly stochastic matrices at the matrix $J_{n}$ (each of whose entries is $1 / n$ ). The conjecture has some interesting interpretations in finite probability and finite combinatorics. However, it is only known to be true for $n$ less than or equal to 5 [6], [1], [2] and for the class of positive semidefinite, hermitian matrices [5]. The most general result previously known was obtained independently by D. London [4] and by M. B. Hedrick [3] and stated that if $A$ is a matrix in $D_{n}$ at which the permanent achieves a local minimum relative to $D_{n}$, then per $A \leqq$ per $A(i \mid j)$ with equality for each positive $a_{i j}$. Both of these papers relied heavily on methods from the definitive work of $\mathbf{M}$. Marcus and M. Newman [6] in which knowledge of the eigenvalues of $A A^{T}$ was required. Since the relationship between the eigenvalues of $A$ or $A A^{T}$ and the permanent of $A$ appears to be extremely nebulous [7], the author finds a great deal of beauty in the simplicity and purely combinatorial nature of the following proof.

THEOREM. Let $A$ be a doubly stochastic matrix, and let $X$ be a set of doubly stochastic matrices with the same $(0,1)$-pattern as $A$ in some neighborhood of $A$. If $A$ is a critical point of the permanent relative to $X$, then per $A=$ per $A(i \mid j)$ for each positive $a_{i j}$.

AMS (MOS) subject classifications (1970). Primary 05B20, 60J05, 15A15, 15A51.

Key words and phrases. Permanent, doubly stochastic matrix, finite combinatorics, finite probability, critical point of the permanent relative to $X$.

${ }^{1}$ This paper is dedicated to Terrie. 
Proof. Assume that for some positive $a_{i j}$, per $A \neq$ per $A(i \mid j)$. Observe that for any fixed $i$, the permanent of $A$ equals the sum of the $a_{i j}$ per $A(i \mid j)$ when one sums on $j$ for positive $a_{i j}$. Thus $\min _{j}$ per $A(i \mid j) \leqq \operatorname{per} A \leqq$ $\max _{j} \operatorname{per}(i \mid j)$ with equality at either extreme if and only if there is equality throughout. A similar remark can be made for fixed $j$.

Let $a_{i(1), j(1)}$ be a positive entry in $A$ at which the per $A(i(1) \mid j(1))$ equals $\max \operatorname{per} A(i \mid j)$ for all positive $a_{i j}$. By the previous remarks, per $A<$ per $A(i(1) \mid j(1))$. Likewise, there is some positive $a_{i(1), j(2)}$ such that per $A(i(1) j(2))$ is less than per $A$. Then there is some positive $a_{i(2), j(2)}$ such that per $A(i(2) \mid j(2))$ is greater than per $A$. Within $n$ times, we must return to a row or a column which has been used previously. For notational convenience, we shall assume that $a_{i(1), j(1)}, a_{i(1), j(2)}, \cdots, a_{i(p), j(p-1)}$, $a_{i(p), j(1)}$ are positive entries of $A$ such that per $A(i(1) \mid j(1))$ is greater than per $A$, per $A(i(1) \mid j(2))$ is less than per $A, \cdots, \operatorname{per} A(i(p) \mid j(p-1))$ is greater than per $A$, and per $A(i(p) \mid j(1))$ is less than per $A$, and the rows and columns which are used occur exactly twice.

Choose $0<x<\min \left\{a_{i(1), j(1)}, \cdots, a_{i(p), j(1)}\right\}$. Consider the doubiy stochastic matrix $A(x)$ defined by $a_{i j}(x)=a_{i j}$ for $(i, j)$ different from $(i(1), j(1)), \cdots$, $(i(p), j(1))$ and

and

$$
\begin{aligned}
a_{i(1), j(1)}(x) & =a_{i(1), j(1)}-x, \\
a_{i(1), j(2)}(x) & =a_{i(1), j(2)}+x, \\
& \cdot \\
& \cdot \\
& \cdot \\
a_{i(p), j(p-1)}(x) & =a_{i(p), j(p-1)}-x,
\end{aligned}
$$

$$
a_{i(p), j(1)}(x)=a_{i(p), j(1)}+x .
$$

Define $f(x)$ to be per $A(x)$. Then since $f(0)$ equals per $A$ and $A$ is a critical point of the permanent relative to $X$, the derivative of $f(x)$ evaluated at 0 equals 0 . Thus

$$
\begin{aligned}
{[\operatorname{per} A(i(1) \mid j(2))+\cdots} & +\operatorname{per} A(i(p) \mid j(1))] \\
& -[\operatorname{per} A(i(1) \mid j(1))+\cdots+\operatorname{per} A(i(p) \mid j(p-1))]
\end{aligned}
$$

equals the derivative of $f(x)$ evaluated at 0 which equals 0 . However, by the choice of the per $A(i(1) \mid j(1)), \cdots$, per $A(i(p) \mid j(1))$, the above sum is negative which is a contradiction.

COROLlary I. If $A$ is a matrix at which the permanent achieves a local minimum relative to $D_{n}$, then per $A \leqq \operatorname{per} A(i \mid j)$ with equality whenever $a_{i j}$ is positive.

The procedure to prove Corollary I can be found in [3]. 
COROllary II. If $A$ is a matrix at which the permanent achieves a local maximum relative to $D_{n}$, then per $A \geqq \operatorname{per} A(i \mid j)$ with equality whenever $a_{i j}$ is positive.

Write $A$ as a direct sum of fully indecomposable matrices and use the techniques in [3].

It is worth noting that the boundary of $D_{n}$ satisfies the hypothesis of the Theorem. Likewise, let $B$ be a doubly stochastic matrix. Define $X$ to be the set of all doubly stochastic matrices $C$ such that $c_{i j}$ is zero whenever $b_{i j}$ is zero. Then $X$ satisfies the hypothesis of the Theorem.

M. Marcus and M. Newman showed that the permanent achieves a local minimum at $J_{n}[6]$, and it is immediate that the permanent achieves an absolute maximum at a permutation matrix (take the product of the $n$ row sums). The author would be very interested in seeing examples at which the permanent achieves a local minimum or local maximum relative to $D_{n}$ besides these.

\section{REFERENCES}

1. P. J. Eberlein, Remarks on the van der Waerden conjecture. II, Linear Algebra and Appl. 2 (1969), 311-320. MR 40 \#160.

2. P. J. Eberlein and G. S. Mudholkar, Some remarks on the van der Waerden conjecture, J. Combinatorial Theory 5 (1968), 386-396. MR 38 \#163.

3. M. B. Hedrick, P-minors of the doubly stochastic matrix at which the permanent achieves a minimum, Linear Algebra and Appl. (to appear).

4. D. London, Some notes on the van der Waerden conjecture, Linear Algebra and Appl. 4 (1971), 155-160. MR 45 \#279.

5. M. Marcus and H. Minc, On a conjecture of B. L. van der Waerden, Proc. Cambridge Philos. Soc. 63 (1967), 305-309. MR 34 \#5853.

6. M. Marcus and M. Newman, On the minimum of the permanent of a doubly stochastic matrix, Duke Math. J. 26 (1959), 61-72. MR 21 \#3432.

7. D. W. Sasser and M. L. Slater, On the inequality $\sum x_{i} y_{j} \geqq(1 / n) \sum x_{i} \sum y_{j}$ and the van der Waerden conjecture, J. Combinatorial Theory 3 (1967), 25-33. MR 36 \#190.

8. B. L. van der Waerden, Aufgabe 45, Jber. Deutsch. Math.-Verein. 35 (1926), 117.

1205 Eastman, Pasadena, Texas 77506

Current address: 222 Kalmer, Pasadena, Texas 77502 